\title{
On the Positive Moments of Ranks of Partitions
}

\author{
William Y.C. Chen ${ }^{1}$, Kathy Q. Ji ${ }^{2}$, and Erin Y.Y. Shen ${ }^{3}$ \\ Center for Combinatorics, LPMC-TJKLC \\ Nankai University \\ Tianjin 300071, P.R. China \\ ${ }^{1}$ chen@nankai.edu.cn, ${ }^{2}$ ji@nankai.edu.cn, ${ }^{3}$ shenyiying@mail.nankai.edu.cn \\ Submitted: Nov 1, 2013; Accepted: Jan 12, 2014; Published: Feb 7, 2014 \\ Mathematics Subject Classifications: 05A17, 11P83, 05A30
}

\begin{abstract}
By introducing $k$-marked Durfee symbols, Andrews found a combinatorial interpretation of the $2 k$-th symmetrized moment $\eta_{2 k}(n)$ of ranks of partitions of $n$ in terms of $(k+1)$-marked Durfee symbols of $n$. In this paper, we consider the $k$-th symmetrized positive moment $\bar{\eta}_{k}(n)$ of ranks of partitions of $n$ which is defined as the truncated sum over positive ranks of partitions of $n$. As combinatorial interpretations of $\bar{\eta}_{2 k}(n)$ and $\bar{\eta}_{2 k-1}(n)$, we show that for given $k$ and $i$ with $1 \leqslant i \leqslant k+1$, $\bar{\eta}_{2 k-1}(n)$ equals the number of $(k+1)$-marked Durfee symbols of $n$ with the $i$-th rank being zero and $\bar{\eta}_{2 k}(n)$ equals the number of $(k+1)$-marked Durfee symbols of $n$ with the $i$-th rank being positive. The interpretations of $\bar{\eta}_{2 k-1}(n)$ and $\bar{\eta}_{2 k}(n)$ are independent of $i$, and they imply the interpretation of $\eta_{2 k}(n)$ given by Andrews since $\eta_{2 k}(n)$ equals $\bar{\eta}_{2 k-1}(n)$ plus twice of $\bar{\eta}_{2 k}(n)$. Moreover, we obtain the generating functions of $\bar{\eta}_{2 k}(n)$ and $\bar{\eta}_{2 k-1}(n)$.
\end{abstract}

Keywords: rank of a partition; $k$-marked Durfee symbol; moment of ranks

\section{Introduction}

This paper is concerned with a combinatorial study of the symmetrized positive moments of ranks of partitions. The notion of symmetrized moments was introduced by Andrews [1]. Any odd symmetrized moment is zero because of the symmetry of ranks. For an even symmetrized moment, Andrews found a combinatorial interpretation by introducing $k$-marked Durfee symbols. It is natural to investigate the combinatorial interpretation of an odd symmetrized moment which is defined as a truncated sum over positive ranks of 
partitions of $n$. We give combinatorial interpretations of both the even and the odd positive moments in terms of $k$-marked Durfee symbols, which also lead to the combinatorial interpretation of an even symmetrized moment of ranks given by Andrews.

The rank of a partition $\lambda$ introduced by Dyson [6] is defined as the largest part minus the number of parts. Let $N(m, n)$ denote the number of partitions of $n$ with rank $m$. The following generating function of $N(m, n)$ was conjectured by Dyson [6] in 1944 and proved by Atkin and Swinnerton-Dyer [3] in 1954. A combinatorial proof was found by Dyson [7] in 1969.

Theorem 1.1. For given integer $m$, we have

$$
\sum_{n=0}^{+\infty} N(m, n) q^{n}=\frac{1}{(q ; q)_{\infty}} \sum_{n=1}^{+\infty}(-1)^{n-1} q^{n(3 n-1) / 2+|m| n}\left(1-q^{n}\right) .
$$

Recently, Andrews [1] introduced the $k$-th symmetrized moment $\eta_{k}(n)$ of ranks of partitions of $n$ as given by

$$
\eta_{k}(n)=\sum_{m=-\infty}^{+\infty}\left(\begin{array}{c}
m+\left\lfloor\frac{k-1}{2}\right\rfloor \\
k
\end{array}\right) N(m, n) .
$$

It can be easily seen that for any $k, \eta_{k}(n)$ is a linear combination of the moments $N_{j}(n)$ of ranks given by Atkin and Garvan [4]

$$
N_{j}(n)=\sum_{m=-\infty}^{\infty} m^{j} N(m, n) .
$$

For example,

$$
\eta_{6}(n)=\frac{1}{720} N_{6}(n)-\frac{1}{144} N_{4}(n)+\frac{1}{180} N_{2}(n) .
$$

In view of the symmetry $N(-m, n)=N(m, n)$, we have $\eta_{2 k+1}(n)=0$. As for an even symmetrized moment $\eta_{2 k}(n)$, Andrews gave the following combinatorial interpretation by introducing $k$-marked Durfee symbols. For the definition of $k$-marked Durfee symbols, see Section 2.

Theorem 1.2 (Andrews [1]). For any $k \geqslant 1, \eta_{2 k}(n)$ is equal to the number of $(k+1)$ marked Durfee symbols of $n$.

Andrews [1] proved the above theorem by using the $k$-fold generalization of Watson's $q$-analog of Whipple's theorem. Ji [9] found a combinatorial proof of Theorem 1.2 by establishing a map from $k$-marked Durfee symbols to ordinary partitions. Kursungoz [10] gave another proof of Theorem 1.2 by using an alternative representation of $k$-marked Durfee symbols.

In this paper, we introduce the $k$-th symmetrized positive moment $\bar{\eta}_{k}(n)$ of ranks as given by

$$
\bar{\eta}_{k}(n)=\sum_{m=1}^{\infty}\left(\begin{array}{c}
m+\left\lfloor\frac{k-1}{2}\right\rfloor \\
k
\end{array}\right) N(m, n)
$$


or equivalently,

$$
\bar{\eta}_{2 k-1}(n)=\sum_{m=1}^{\infty}\left(\begin{array}{c}
m+k-1 \\
2 k-1
\end{array}\right) N(m, n)
$$

and

$$
\bar{\eta}_{2 k}(n)=\sum_{m=1}^{\infty}\left(\begin{array}{c}
m+k-1 \\
2 k
\end{array}\right) N(m, n) .
$$

Furthermore, it is easy to see that for any $k, \bar{\eta}_{k}(n)$ is a linear combination of the positive moments $\bar{N}_{j}(n)$ of ranks introduced by Andrews, Chan and Kim [2] as given by

$$
\bar{N}_{j}(n)=\sum_{m=1}^{\infty} m^{j} N(m, n) .
$$

For example,

$$
\begin{aligned}
& \bar{\eta}_{4}(n)=\frac{1}{24} \bar{N}_{4}(n)-\frac{1}{12} \bar{N}_{3}(n)-\frac{1}{24} \bar{N}_{2}(n)+\frac{1}{12} \bar{N}_{1}(n), \\
& \bar{\eta}_{5}(n)=\frac{1}{120} \bar{N}_{5}(n)-\frac{1}{24} \bar{N}_{3}(n)+\frac{1}{30} \bar{N}_{1}(n) .
\end{aligned}
$$

By the symmetry $N(-m, n)=N(m, n)$, it is readily seen that

$$
\eta_{2 k}(n)=2 \bar{\eta}_{2 k}(n)+\bar{\eta}_{2 k-1}(n) .
$$

The main objective of this paper is to give combinatorial interpretations of $\bar{\eta}_{2 k}(n)$ and $\bar{\eta}_{2 k-1}(n)$. We show that for given $k$ and $i$ with $1 \leqslant i \leqslant k+1, \bar{\eta}_{2 k-1}(n)$ equals the number of $(k+1)$-marked Durfee symbols of $n$ with the $i$-th rank being zero and $\bar{\eta}_{2 k}(n)$ equals the number of $(k+1)$-marked Durfee symbols of $n$ with the $i$-th rank being positive. It should be noted that $\bar{\eta}_{2 k-1}(n)$ and $\bar{\eta}_{2 k}(n)$ are independent of $i$ since the ranks of $k$-marked Durfee symbols are symmetric, see Andrews [1, Corollary 12].

With the aid of Theorem 2.1 and Theorem 2.2 together with the generating function (1.1) of $N(m, n)$, we obtain the generating functions of $\bar{\eta}_{2 k}(n)$ and $\bar{\eta}_{2 k-1}(n)$.

\section{Combinatorial interpretations}

In this section, we give combinatorial interpretations of $\bar{\eta}_{2 k-1}(n)$ and $\bar{\eta}_{2 k}(n)$ in terms of $k$-marked Durfee symbols. For a partition $\lambda$ of $n$, we write $\lambda=\left(\lambda_{1}, \lambda_{2}, \ldots, \lambda_{s}\right)$ with the entries $\lambda_{i}$ in nonincreasing order such that $\lambda_{1}+\lambda_{2}+\cdots+\lambda_{s}=n$. We assume that all the parts of $\lambda$ are positive. The number of parts of $\lambda$ is called the length of $\lambda$, denoted by $\ell(\lambda)$. The weight of $\lambda$ is the sum of parts, denoted $|\lambda|$.

Recall that a $k$-marked Durfee symbol of $n$ introduced by Andrews [1] is a two-line array composed of $k$ pairs of partitions $\left(\alpha^{1}, \beta^{1}\right),\left(\alpha^{2}, \beta^{2}\right), \ldots,\left(\alpha^{k}, \beta^{k}\right)$ along with a positive integer $D$ which is represented in the following form:

$$
\tau=\left(\begin{array}{cccc}
\alpha^{k}, & \alpha^{k-1}, & \ldots, & \alpha^{1} \\
\beta^{k}, & \beta^{k-1}, & \ldots, & \beta^{1}
\end{array}\right)_{D},
$$


where the partitions $\alpha^{i}=\left(\alpha_{1}^{i}, \alpha_{2}^{i}, \ldots, \alpha_{s}^{i}\right)$ and $\beta^{i}=\left(\beta_{1}^{i}, \beta_{2}^{i}, \ldots, \beta_{s}^{i}\right)$ satisfy the following four conditions:

(1) The partitions $\alpha^{i}(1 \leqslant i<k)$ are nonempty, while $\alpha^{k}$ and $\beta^{i}(1 \leqslant i \leqslant k)$ are allowed to be empty;

(2) $\beta_{1}^{i-1} \leqslant \alpha_{1}^{i-1} \leqslant \min \left\{\alpha_{s}^{i}, \beta_{s}^{i}\right\}$ for $2 \leqslant i \leqslant k$;

(3) $\alpha_{1}^{k}, \beta_{1}^{k} \leqslant D$;

(4) $\sum_{i=1}^{k}\left(\left|\alpha^{i}\right|+\left|\beta^{i}\right|\right)+D^{2}=n$.

Let

$$
\tau=\left(\begin{array}{cccc}
\alpha^{k}, & \alpha^{k-1}, & \ldots, & \alpha^{1} \\
\beta^{k}, & \beta^{k-1}, & \ldots, & \beta^{1}
\end{array}\right)_{D}
$$

be a $k$-marked Durfee symbol. The pair $\left(\alpha^{i}, \beta^{i}\right)$ of partitions is called the $i$-th vector of $\tau$. Andrews defined the $i$-th $\operatorname{rank} \rho_{i}(\tau)$ of $\tau$ as follows

$$
\rho_{i}(\tau)= \begin{cases}\ell\left(\alpha^{i}\right)-\ell\left(\beta^{i}\right)-1, & \text { for } 1 \leqslant i<k, \\ \ell\left(\alpha^{k}\right)-\ell\left(\beta^{k}\right), & \text { for } i=k .\end{cases}
$$

For example, consider the following 3-marked Durfee symbol

$$
\tau=\left(\begin{array}{ccc}
\overbrace{5_{3}, 4_{3}}^{\alpha^{3}}, & \overbrace{4_{2}, 3_{2}, 3_{2}, 2_{2}}^{\alpha^{2}}, & \overbrace{2_{1}}^{\alpha^{1}} \\
\underbrace{4_{3}}_{\beta^{3}}, & \underbrace{3_{2}, 2_{2}, 2_{2}}_{\beta^{2}}, & \underbrace{2_{1}, 2_{1}}_{\beta^{1}}
\end{array}\right)_{5} .
$$

We have $\rho_{1}(\tau)=-2, \rho_{2}(\tau)=0$, and $\rho_{3}(\tau)=1$.

For an odd symmetrized moment $\bar{\eta}_{2 k-1}(n)$, we have the following combinatorial interpretation.

Theorem 2.1. For given positive integers $k$ and $i$ with $1 \leqslant i \leqslant k+1, \bar{\eta}_{2 k-1}(n)$ is equal to the number of $(k+1)$-marked Durfee symbols of $n$ with the $i$-th rank equal to zero.

For the even case, we have the following interpretation.

Theorem 2.2. For given positive integers $k$ and $i$ with $1 \leqslant i \leqslant k+1, \bar{\eta}_{2 k}(n)$ is equal to the number of $(k+1)$-marked Durfee symbols of $n$ with the $i$-th rank being positive.

The proofs of the above two interpretations are based on the following partition identity obtained by Ji [9]. We shall adopt the notation $D_{k}\left(m_{1}, m_{2}, \ldots, m_{k} ; n\right)$ as used by Andrews [1] to denote the number of $k$-marked Durfee symbols of $n$ with the $i$-th rank equal to $m_{i}$ for $1 \leqslant i \leqslant k$. 
Theorem 2.3. For $k \geqslant 2$ and $n \geqslant 1$, we have

$$
D_{k}\left(m_{1}, \ldots, m_{k} ; n\right)=\sum_{t_{1}, \ldots, t_{k-1}=0}^{\infty} N\left(\sum_{i=1}^{k}\left|m_{i}\right|+2 \sum_{i=1}^{k-1} t_{i}+k-1, n\right) .
$$

To derive the above interpretations of $\bar{\eta}_{2 k-1}(n)$ and $\bar{\eta}_{2 k}(n)$, we also need the following symmetric property given by Andrews [1]. Boulet and Kursungoz [5] found a combinatorial proof of this fact.

Theorem 2.4. For $k \geqslant 2$ and $n \geqslant 1, D_{k}\left(m_{1}, \ldots, m_{k} ; n\right)$ is symmetric in $m_{1}, m_{2}, \ldots, m_{k}$.

We are now in a position to prove Theorem 2.1 and Theorem 2.2. Proof of Theorem 2.1. By Theorem 2.4, it suffices to show that

$$
\sum_{m_{2}, m_{3}, \ldots, m_{k+1}=-\infty}^{\infty} D_{k+1}\left(0, m_{2}, m_{3}, \ldots, m_{k+1} ; n\right)=\bar{\eta}_{2 k-1}(n) .
$$

Using Theorem 2.3, we get

$$
\begin{aligned}
\sum_{m_{2}, m_{3}, \ldots, m_{k+1}}^{\infty} & D_{k+1}\left(0, m_{2}, m_{3}, \ldots, m_{k+1} ; n\right) \\
= & \sum_{m_{2}, m_{3}, \ldots, m_{k+1}=-\infty}^{\infty} \sum_{t_{1}, \ldots, t_{k}=0}^{\infty} N\left(\sum_{i=2}^{k+1}\left|m_{i}\right|+2 \sum_{i=1}^{k} t_{i}+k, n\right) .
\end{aligned}
$$

For $k \geqslant 1$ and $m \geqslant k$, let $c_{k}(m)$ denote the number of integer solutions to the equation

$$
\left|m_{2}\right|+\cdots+\left|m_{k+1}\right|+2 t_{1}+\cdots+2 t_{k}=m-k,
$$

where $m_{i}$ are integers and $t_{i}$ are nonnegative integers. It is easy to see that the generating function of $c_{k}(m)$ is equal to

$$
\begin{aligned}
\sum_{m=k}^{\infty} c_{k}(m) q^{m-k} & =\left(1+2 q+2 q^{2}+2 q^{3}+\cdots\right)^{k}\left(1+q^{2}+q^{4}+q^{6}+\cdots\right)^{k} \\
& =\left(\frac{1+q}{1-q}\right)^{k}\left(\frac{1}{1-q^{2}}\right)^{k} \\
& =\frac{1}{(1-q)^{2 k}} \\
& =\sum_{m=k}^{\infty}\left(\begin{array}{c}
m+k-1 \\
2 k-1
\end{array}\right) q^{m-k} .
\end{aligned}
$$

Hence

$$
c_{k}(m)=\left(\begin{array}{c}
m+k-1 \\
2 k-1
\end{array}\right)
$$


and (2.3) can be written as

$$
\begin{gathered}
\sum_{m_{2}, m_{3}, \ldots, m_{k+1}=-\infty}^{\infty} D_{k+1}\left(0, m_{2}, m_{3}, \ldots, m_{k+1} ; n\right) \\
=\sum_{m=1}^{\infty}\left(\begin{array}{c}
m+k-1 \\
2 k-1
\end{array}\right) N(m, n),
\end{gathered}
$$

which is the defining expression of $\bar{\eta}_{2 k-1}(n)$. This completes the proof.

Proof of Theorem 2.2. Similarly, by Theorem 2.4, it is sufficient to show that

$$
\sum_{\substack{m_{1}>0 \\ m_{2}, m_{3}, \ldots, m_{k+1}=-\infty}}^{\infty} D_{k+1}\left(m_{1}, m_{2}, \ldots, m_{k+1} ; n\right)=\bar{\eta}_{2 k}(n) .
$$

Invoking Theorem 2.3, we get

$$
\begin{aligned}
\sum_{\substack{m_{1}>0 \\
m_{2}, m_{3}, \ldots, m_{k+1}=-\infty}}^{\infty} D_{k+1}\left(m_{1}, m_{2}, \ldots, m_{k+1} ; n\right) & \\
= & \sum_{\substack{m_{1}>0 \\
m_{2}, m_{3}, \ldots, m_{k+1}=-\infty}}^{\infty} \sum_{t_{1}, \ldots, t_{k}=0}^{\infty} N\left(m_{1}+\sum_{i=2}^{k+1}\left|m_{i}\right|+2 \sum_{i=1}^{k} t_{i}+k, n\right) .
\end{aligned}
$$

For $k \geqslant 1$ and $m \geqslant k+1$, let $\bar{c}_{k}(m)$ denote the number of integer solutions to the equation

$$
m_{1}+\left|m_{2}\right|+\cdots+\left|m_{k+1}\right|+2 t_{1}+\cdots+2 t_{k}=m-k,
$$

where $m_{1}$ is a positive integer, $m_{i}(2 \leqslant i \leqslant k+1)$ are integers and $t_{i}$ are nonnegative integers. An easy computation shows that

$$
\sum_{m=k+1}^{\infty} \bar{c}_{k}(m) q^{m-k}=\frac{q}{(1-q)^{2 k+1}}
$$

so that

$$
\bar{c}_{k}(m)=\left(\begin{array}{c}
m+k-1 \\
2 k
\end{array}\right) .
$$

Thus, the sum on the right hand side of (2.6) becomes

$$
\sum_{m=1}^{\infty}\left(\begin{array}{c}
m+k-1 \\
2 k
\end{array}\right) N(m, n)
$$

which is in accordance with the definition of $\bar{\eta}_{2 k}(n)$, and hence the proof is complete.

Note that the number $D_{k}\left(m_{1}, \ldots, m_{k} ; n\right)$ has the mirror symmetry with respect to each $m_{i}$, that is, for $1 \leqslant i \leqslant k$, we have

$$
D_{k}\left(m_{1}, \ldots, m_{i}, \ldots, m_{k} ; n\right)=D_{k}\left(m_{1}, \ldots,-m_{i}, \ldots, m_{k} ; n\right) .
$$

Using this symmetry property, Theorem 2.2 can be restated as follows. 
Theorem 2.5. For given positive integers $k$ and $i$ with $1 \leqslant i \leqslant k+1, \bar{\eta}_{2 k}(n)$ is also equal to the number of $(k+1)$-marked Durfee symbols of $n$ with the $i$-th rank being negative.

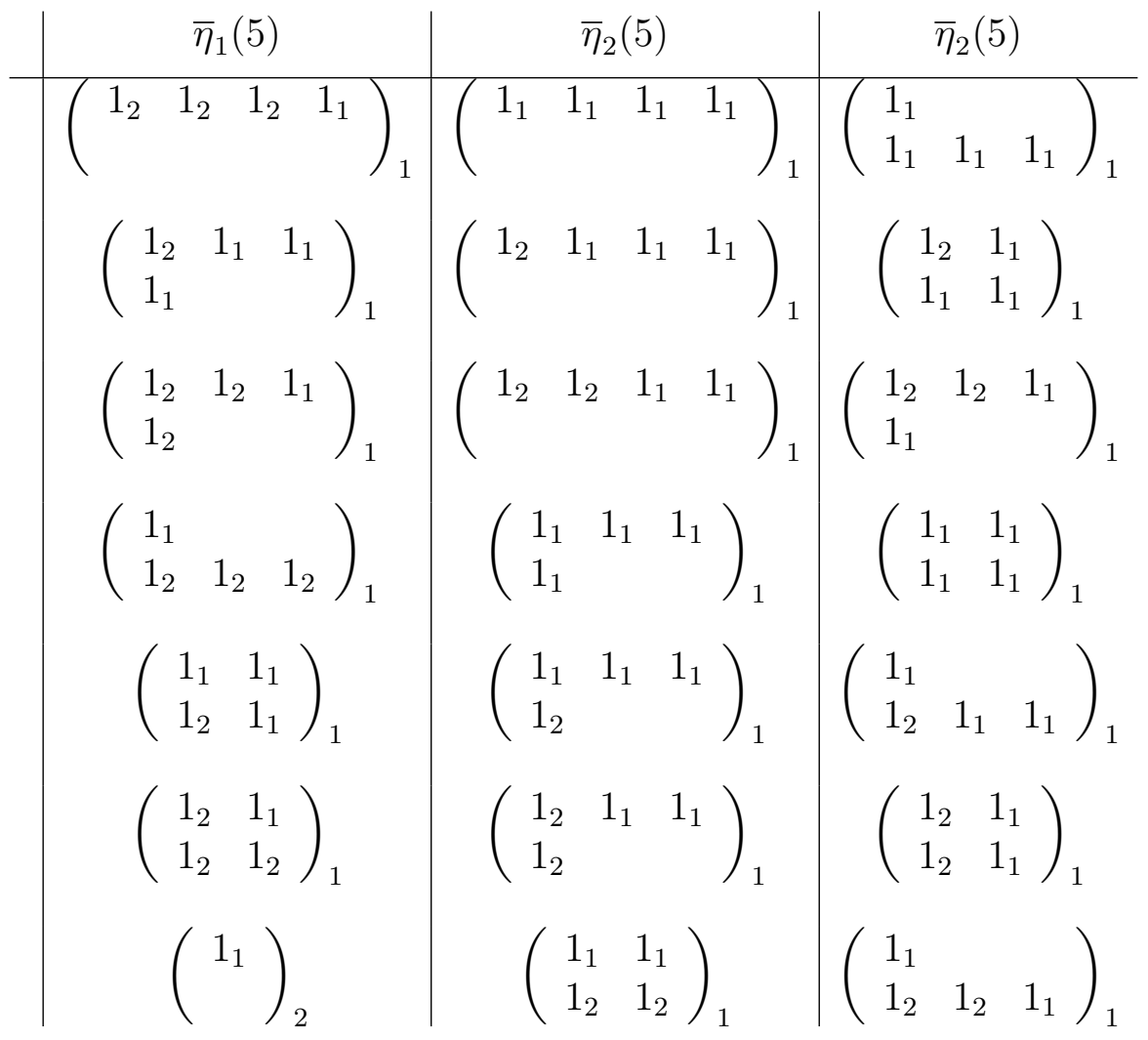

Table 2.1: 2-Marked Durfee Symbols of 5.

For example, for $n=5, k=1$ and $i=1$, there are twenty-one 2-marked Durfee symbols of 5 as listed in Table 2.1. The first column in Table 2.1 gives seven 2-marked Durfee symbols $\tau$ with $\rho_{1}(\tau)=0$, the second column contains seven 2-marked Durfee symbols $\tau$ with $\rho_{1}(\tau)>0$ and the third column contains seven 2-marked Durfee symbols $\tau$ with $\rho_{1}(\tau)<0$. It can be verified that $\bar{\eta}_{1}(5)=7, \bar{\eta}_{2}(5)=7$ and $\eta_{2}(5)=\bar{\eta}_{1}(5)+2 \bar{\eta}_{2}(5)=21$.

\section{The generating functions of $\bar{\eta}_{2 k-1}(n)$ and $\bar{\eta}_{2 k}(n)$}

In this section, we obtain the generating functions of $\bar{\eta}_{2 k-1}(n)$ and $\bar{\eta}_{2 k}(n)$ with the aid of Theorem 2.1 and Theorem 2.2. In doing so, we use the generating function of $N(m, n)$ to derive the generating functions of $D_{k+1}\left(0, m_{2}, \ldots, m_{k+1} ; n\right)$ and $D_{k+1}\left(m_{1}, m_{2}, \ldots, m_{k+1} ; n\right)$. 
Theorem 3.1. For $k \geqslant 1$, we have

$$
\begin{aligned}
& \sum_{m_{2}, \ldots, m_{k+1}=-\infty}^{\infty} \sum_{n=0}^{\infty} D_{k+1}\left(0, m_{2}, \ldots, m_{k+1} ; n\right) x_{1}^{m_{2}} \cdots x_{k}^{m_{k+1}} q^{n} \\
& =\frac{1}{(q ; q)_{\infty}} \sum_{n=1}^{\infty}(-1)^{n-1} q^{n(3 n-1) / 2+k n} \frac{\left(1-q^{n}\right)}{\prod_{j=1}^{k}\left(1-x_{j} q^{n}\right)\left(1-x_{j}^{-1} q^{n}\right)}
\end{aligned}
$$

Proof. Let

$$
G_{k}\left(x_{1}, \ldots, x_{k} ; q\right)=\sum_{m_{2}, \ldots, m_{k+1}=-\infty}^{\infty} \sum_{n=0}^{\infty} D_{k+1}\left(0, m_{2}, \ldots, m_{k+1} ; n\right) x_{1}^{m_{2}} \cdots x_{k}^{m_{k+1}} q^{n} .
$$

By Theorem 2.3, we have

$$
\begin{aligned}
& G_{k}\left(x_{1}, \ldots, x_{k} ; q\right) \\
& \quad=\sum_{m_{2}, \ldots, m_{k+1}=-\infty}^{\infty} \sum_{t_{1}, \ldots, t_{k}=0}^{\infty} x_{1}^{m_{2}} \cdots x_{k}^{m_{k+1}} \sum_{n=0}^{\infty} N\left(\sum_{i=2}^{k+1}\left|m_{i}\right|+2 \sum_{i=1}^{k} t_{i}+k, n\right) q^{n} .
\end{aligned}
$$

Using the generating function (1.1) of $N(m, n)$ with $m$ replaced by $\sum_{i=2}^{k+1}\left|m_{i}\right|+2 \sum_{i=1}^{k} t_{i}+$ $k$, we find that

$$
\begin{aligned}
\sum_{n=0}^{\infty} N & \left(\sum_{i=2}^{k+1}\left|m_{i}\right|+2 \sum_{i=1}^{k} t_{i}+k, n\right) q^{n} \\
& =\frac{1}{(q ; q)_{\infty}} \sum_{n=1}^{\infty}(-1)^{n-1} q^{n(3 n-1) / 2+n\left(\sum_{i=2}^{k+1}\left|m_{i}\right|+2 \sum_{i=1}^{k} t_{i}+k\right)}\left(1-q^{n}\right) .
\end{aligned}
$$

Substituting (3.3) into (3.2), we get

$$
\begin{aligned}
G_{k}\left(x_{1}, \ldots, x_{k} ; q\right)= & \sum_{m_{2}, \ldots, m_{k+1}=-\infty}^{\infty} \sum_{t_{1}, \ldots, t_{k}=0}^{\infty} x_{1}^{m_{2}} \cdots x_{k}^{m_{k+1}} \\
& \times \frac{1}{(q ; q)_{\infty}} \sum_{n=1}^{\infty}(-1)^{n-1} q^{n(3 n-1) / 2+n\left(\sum_{i=2}^{k+1}\left|m_{i}\right|+2 \sum_{i=1}^{k} t_{i}+k\right)}\left(1-q^{n}\right) .
\end{aligned}
$$

Write (3.4) in the following form

$$
\begin{aligned}
G_{k}\left(x_{1}, \ldots, x_{k} ; q\right)= & \frac{1}{(q ; q)_{\infty}} \sum_{n=1}^{\infty}(-1)^{n-1} q^{n(3 n-1) / 2+k n}\left(1-q^{n}\right) \\
& \times \sum_{m_{2}, \ldots, m_{k+1}=-\infty}^{\infty} \sum_{t_{1}, \ldots, t_{k}=0}^{\infty} x_{1}^{m_{2}} \cdots x_{k}^{m_{k+1}} q^{n\left(\sum_{i=2}^{k+1}\left|m_{i}\right|+2 \sum_{i=1}^{k} t_{i}\right)} .
\end{aligned}
$$


Notice that

$$
\sum_{a=-\infty}^{+\infty} \sum_{b=0}^{+\infty} x^{a} q^{n(|a|+2 b)}=\frac{1}{\left(1-x q^{n}\right)\left(1-x^{-1} q^{n}\right)}
$$

Applying the above formula (3.6) repeatedly to (3.5), we deduce that

$$
G_{k}\left(x_{1}, \ldots, x_{k} ; q\right)=\frac{1}{(q ; q)_{\infty}} \sum_{n=1}^{\infty}(-1)^{n-1} q^{n(3 n-1) / 2+k n} \frac{\left(1-q^{n}\right)}{\prod_{j=1}^{k}\left(1-x_{j} q^{n}\right)\left(1-x_{j}^{-1} q^{n}\right)},
$$

as required.

Setting $x_{j}=1$ for $1 \leqslant j \leqslant k$ in Theorem 3.1 and applying Theorem 2.1, we arrive at the following generating function of $\bar{\eta}_{2 k-1}(n)$.

Corollary 3.2. For $k \geqslant 1$, we have

$$
\sum_{n=1}^{\infty} \bar{\eta}_{2 k-1}(n) q^{n}=\frac{1}{(q ; q)_{\infty}} \sum_{n=1}^{\infty}(-1)^{n-1} q^{n(3 n-1) / 2+k n} \frac{1}{\left(1-q^{n}\right)^{2 k-1}} .
$$

Since $\bar{\eta}_{1}(n)=\bar{N}_{1}(n)$, when taking $k=1$ in (3.7), we are led to the generating function for $\bar{N}_{1}(n)$ as given by Andrews, Chan and Kim in [2, Theorem 1].

The following generating function can be derived by using the same reasoning as in the proof of Theorem 3.1.

Theorem 3.3. For $k \geqslant 1$, we have

$$
\begin{aligned}
& \sum_{\substack{m_{1}>0 \\
m_{2}, \ldots, m_{k+1}=-\infty}}^{\infty} \sum_{n=1}^{\infty} D_{k+1}\left(m_{1}, m_{2}, \ldots, m_{k+1} ; n\right) x_{1}^{m_{1}} \cdots x_{k+1}^{m_{k+1}} q^{n} \\
= & \frac{1}{(q ; q)_{\infty}} \sum_{n=1}^{\infty}(-1)^{n-1} q^{n(3 n+1) / 2+k n} \frac{x_{1}\left(1-q^{n}\right)}{\left(1-x_{1} q^{n}\right) \prod_{j=2}^{k+1}\left(1-x_{j} q^{n}\right)\left(1-x_{j}^{-1} q^{n}\right)} .
\end{aligned}
$$

Setting $x_{j}=1$ for $1 \leqslant j \leqslant k+1$ in Theorem 3.3 and using Theorem 2.2, we come to the following generating function of $\bar{\eta}_{2 k}(n)$.

Corollary 3.4. For $k \geqslant 1$, we have

$$
\sum_{n=1}^{\infty} \bar{\eta}_{2 k}(n) q^{n}=\frac{1}{(q ; q)_{\infty}} \sum_{n=1}^{\infty}(-1)^{n-1} q^{n(3 n+1) / 2+k n} \frac{1}{\left(1-q^{n}\right)^{2 k}} .
$$

Acknowledgments. We wish to thank the referee for helpful suggestions. This work was supported by the 973 Project and the National Science Foundation of China. 


\section{References}

[1] G. E. Andrews, Partitions, Durfee symbols, and the Atkin-Garvan moments of ranks, Invent. Math. 169 (2007) 37-73.

[2] G. E. Andrews, S. H. Chan, and B. Kim, The odd moments of ranks and cranks, J. Combin. Theory Ser. A 120 (2013) 77-91.

[3] A. O. L. Atkin and P. Swinnerton-Dyer, Some properties of partitions, Proc. London Math. Soc. III. Ser. 4 (1954) 84-106.

[4] A. O. L. Atkin and F. Garvan, Relations between the ranks and cranks of partitions, Ramanujan J. 7 (2003) 343-366.

[5] C. Boulet and K. Kursungoz, Symmetry of $k$-marked Durfee symbols, Int. J. Number Theory 7 (2011) 215-230.

[6] F. Dyson, Some guesses in the theory of partitions, Eureka (Cambridge) 8 (1944) $10-15$.

[7] F. Dyson, A new symmetry of partitions, J. Combin. Theory Ser. A 7 (1969) 56-61.

[8] F. Garvan, New combinatorial interpretations of Ramanujan's partition congruences $\bmod 5,7,11$, Trans. Amer. Math. Soc. 305 (1988) 47-77.

[9] K. Q. Ji, The combinatorics of $k$-marked Durfee symbols, Trans. Amer. Math. Soc. 363 (2011) 987-1005.

[10] K. Kurşungöz, Counting k-marked Durfee symbols, Electron. J. Combin. 18(1) (2011) \#P41. 